Western University

Scholarship@Western

Chemistry Publications

Chemistry Department

Winter 4-20-2018

\title{
Plasmon-Mediated Drilling in Thin Metallic Nanostructures
}

Francois Lagugne-Labarthet

University of Western Ontario, flagugne@uwo.ca

D. M. MRae

University of Western Ontario

K.Jeon

University of Western Ontario

Follow this and additional works at: https://ir.lib.uwo.ca/chempub

Cart of the Chemistry Commons

Citation of this paper:

Lagugne-Labarthet, Francois; MRae, D. M.; and Jeon, K., "Plasmon-Mediated Drilling in Thin Metallic Nanostructures" (2018). Chemistry Publications. 101.

https://ir.lib.uwo.ca/chempub/101 


\section{Plasmon-Mediated Drilling in Thin Metallic}

\section{Nanostructures}

Danielle M. McRae ${ }^{\dagger}$, Keuna Jeon", François Lagugné-Labarthet ${ }^{* \dagger}$

$\dagger$ Department of Chemistry, University of Western Ontario, 1151 Richmond Street, London, Ontario, N6A 5B7, Canada.

KEYWORDS Tip-enhanced Raman, Plasmonics, Finite-element method, Plasmon-mediated reactions, Hot electron transfer

ABSTRACT Thin and ultraflat conductive surfaces are of particular interest to use as substrates for tip-enhanced spectroscopy applications. Tip-enhanced spectroscopy exploits the excitation of a localized surface plasmon resonance mode at the apex of a metallized atomic force microscope tip, confining and enhancing the local electromagnetic field by several orders of magnitude. This allows for nanoscale mapping of the surface with high spatial resolution and surface sensitivity, as demonstrated when coupled to local Raman measurements. In gap-mode tip-enhanced spectroscopy, the specimen of interest is deposited onto a flat metallic surface and probed by a metallic tip, allowing for further electromagnetic confinement and subsequent enhancement. We investigate here a geometry where a gold tip is used in conjunction with a silver nanoplate, thus forming a heterometallic platform for local enhancement. When irradiated, a plasmon-mediated reaction is triggered at the tip-substrate junction due to the enhanced electric field and the transfer 
of hot electrons from the tip to the nanoplate. This resulting nanoscale reaction appears to be sufficient to ablate the thin silver plates even under weak laser intensity. Such an approach may be further exploited for patterning metallic nanostructures or photoinduced chemical reactions at metal surfaces.

\section{INTRODUCTION}

Localized surface plasmon modes are oscillations of conductive electrons that can be observed in nanostructures when using a selected set of wavelengths that match the plasmon resonances. These resonances are primarily sensitive to the material and dimensions of the considered conductive nanostructure, as well as the optical properties of the impinging electromagnetic field, such as energy and polarization. In isolated and sharp-cornered nanostructures, the excitation of plasmon modes generally results in the confinement and enhancement of the local electromagnetic field by several orders of magnitude, and is strongly dependent on the opto-geometric experimental conditions. This locally enhanced electromagnetic field is often exploited for improving the sensitivity of vibrational spectroscopy measurements, such as in surface-enhanced Raman spectroscopy (SERS), ${ }^{1-3}$ and surface-enhanced infrared absorption. ${ }^{4-6}$

Similar to SERS, tip-enhanced Raman spectroscopy (TERS) exploits such localized surface plasmon resonance confined at the apex of a sharp metallic atomic force microscope tip. This confined electromagnetic field is enhanced up to several orders of magnitude and enables significant intensity increase of local optical processes such as Raman scattering. TERS offers both an enhanced signal and spatial resolution beyond the diffraction limit of light, as well as an improved Raman surface sensitivity that can be exploited to probe in situ a variety of surface reactions and understand fundamental electron transfer processes. ${ }^{7}$ 
Further signal enhancement is achieved when the analyte is placed on a metallic substrate, thus forming a nanoscale metallic junction. This mode is referred to as gap-mode TERS, and has been used to probe a variety of molecular systems such as small molecules, ${ }^{8-12}$ nanostructured materials and biomaterials. ${ }^{13-21}$ Ideal metallic substrates for gap-mode TERS in the backscattering configuration are both atomically flat, reducing parasitic scattering processes, and thin enough to be optically transparent. ${ }^{9,} 19,22-23$ For this purpose, previous studies have focused on the use of gold nanoplates as substrates, as gold nanoplates exhibit these attributes and are chemically stable. ${ }^{9}$ 12, 17-19, 21-22 Moreover, the use of silver nanoplates together with silver tips has also been marginally reported, presumably due to the poor chemical stability of silver. ${ }^{15-16}$

Importantly, the plasmon resonance of the coated metallic tip can also be exploited to drive chemical reactions at the nanoscale. Plasmons may decay radiatively, by emission of a photon, or non-radiatively, through the production of a highly energetic "hot" electron. ${ }^{24-25}$ Plasmonmediated reactions are those that are triggered by the combination of the locally enhanced electric field and the presence of hot electrons that are the result of a plasmon resonance. The simultaneous initiation and tracking of these plasmon-mediated reactions is possible through the use of spectroscopic methods such as SERS and TERS.

The dimerization of 4-aminothiophenol or 4-nitrothiophenol to dimercaptoazobenzene is an example of a well investigated plasmon-mediated reaction by SERS. ${ }^{26-30}$ Other plasmon-mediated reactions investigated include polymerization, ${ }^{31-34}$ demethylation of methylene blue, ${ }^{35}$ and water splitting, ${ }^{36-38}$ among others. Hot electron processes have also been investigated for the reduction of aqueous silver and platinum ions. ${ }^{39-40}$ While SERS is effective for monitoring plasmonmediated reactions, it lacks the spatial resolution to observe and control the reaction at the nanometer level. In TERS, since the position of the tip is precisely controlled with sub-nanometer 
resolution, the application of TERS to plasmon-mediated reactions allows for spatially resolved surface reactions and characterization of localized reactions. ${ }^{9,}, 12,31,41-42$

In this work, we perform a plasmon-mediated reaction in a gap-mode configuration, with a heterometallic junction formed by a gold tip and a silver nanoplate. To the best of our knowledge this is the first time that this configuration of metal selection for the tip (gold) and the substrate (silver) is reported. It is shown that, as a result of the plasmon-mediated reaction, the silver nanoplate is locally ablated, as a series of holes appear on the metal surface. Herein, we investigated the possible origin of such plasmon-mediated damage in metallic nanoplates using weak irradiance conditions.

\section{RESULTS AND DISCUSSION}

\section{Characterization of Ag Nanoplates}

For gap-mode TERS experiments using an inverted optical microscope, the metallic nanoplate should exhibit a high width/thickness aspect ratio with minimal surface roughness. Large lateral dimensions of the nanoplates, on the order of several microns, facilitate their observation under an optical microscope, whilst being thin enough for the laser to penetrate through the nanoplate and reach the metal tip. Protocols for the synthesis of gold nanoplates that meet these requirements have been well established. ${ }^{43-44}$ Conversely, the synthetic approaches to preparing silver nanoplates often yield smaller nanoplates with side lengths less than $500 \mathrm{~nm}$, making them less ideal for gap-mode TERS applications. In this study, silver nanoplates were synthesized based on a seed-mediated method developed by Zhang et al. ${ }^{45}$ As can be seen in the scanning electron microscopy (SEM) image of Figure 1a, the resulting nanoplates of the seed-mediated synthesis are large, with a distribution in size ranging between two to six microns in side length. The prepared 
silver nanoplates were commonly found to be triangular or truncated triangles, as shown in Figure 1a. To determine the thickness of the nanoplates, atomic force microscopy (AFM) scans were performed. The inset of Figure 1a shows a representative AFM image of a silver nanoplate with the corresponding cross-section in Figure 1b. Here the thickness of the nanoplates was found to vary from $40-60 \mathrm{~nm}$, and that the surface exhibits an RMS roughness on the order of several nanometers, compared to about $500 \mathrm{pm}$ for gold nanoplates. ${ }^{9}$ The as-prepared nanoplates often stack (Figure 1a), or have smaller nanostructures, such as multifaceted nanoparticles, on their surfaces, but clean, isolated silver plates can be found and used for the TERS experiments.

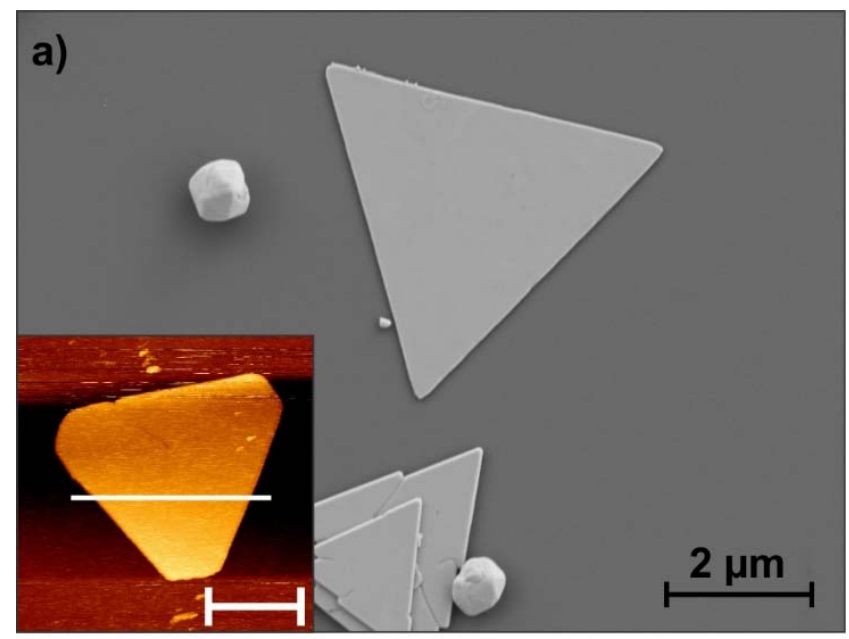

b)

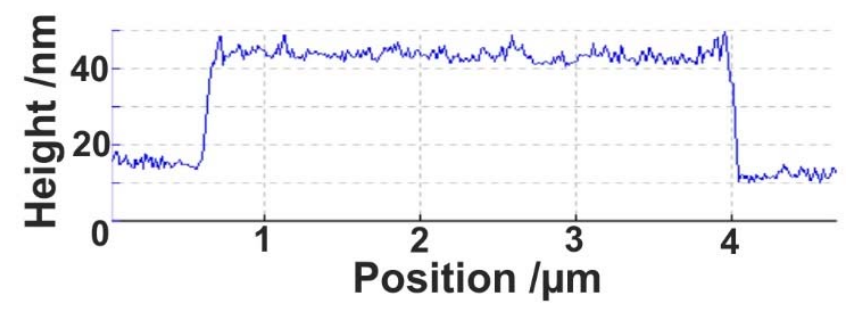

Figure 1: a) SEM of silver nanoplates, with representative atomic force micrograph inset. Scale bar in inset is $2 \mu \mathrm{m}$. b) Cross-section of nanoplate along white line as indicated in the inset of a).

Tip-Enhanced Plasmon Drilling of Ag Nanoplates 
Prior to performing tip-enhanced measurements, the surface of the nanoplate was functionalized with a monolayer of 4-mercaptophenylboronic acid (4-MPBA). Figure 2a shows an AFM scan of a functionalized nanoplate with an intrinsically rough surface, but without other particles or plates present. The nanoplates as synthesized do provide SERS activity, as shown in Figure 2b, where the SERS spectrum has been multiplied by 10 . This is likely due to the rough surface of the nanoplates, as the nanoscale roughness can support hot-spots, even though an irradiation at 632.8 $\mathrm{nm}$ was used, which is generally off-resonance from the plasmon modes observed in silver-based materials. As a linearly polarized laser was used in an axial geometry, the tip was localized slightly off-center of the focused laser beam to benefit from the $z$-component of the tightly focused beam. To collect the TERS measurements, the tip was approached to the centre of the triangular nanoplate to minimize any effects from the edges of the nanoplates. To differentiate the TERS and SERS contributions, a collection of spectra was collected at increasing tip-sample distances, from $0 \mathrm{~nm}$ (tip engaged) to $500 \mathrm{~nm}$ with varying step sizes, and the results are shown in Figure 2b. When the tip is at the surface of the nanoplate, the electromagnetic enhancement offered by the tip is able to locally enhance the Raman spectrum of the 4-MPBA molecules adsorbed on the Ag nanoplate. As the tip is retracted from the surface, the signal decays, but not completely. Instead, the signal from a tip retracted $500 \mathrm{~nm}$ above the nanoplate surface is more intense than the reference SERS spectrum collected on flat nanoplates without any TERS tip. To ascertain the origin of this increase in signal, a post-TERS AFM scan was performed (Figure 2c). The scan clearly shows that the center of the nanoplate has been "drilled", with considerable additional roughness having been introduced around the ablated area. This deformation has amplified the SERS effect through an increase of the localized roughness, as shown in the AFM topography (Figure 2c). To determine if this effect is facilitated by the presence of an adsorbed molecule, the same process was repeated 
on a bare nanoplate (Figure 3a). Once again, after the tip-enhanced measurements were performed, deformation of the nanoplate was observed, and as shown in Figure 3b, is sufficiently large to be seen optically under a microscope.
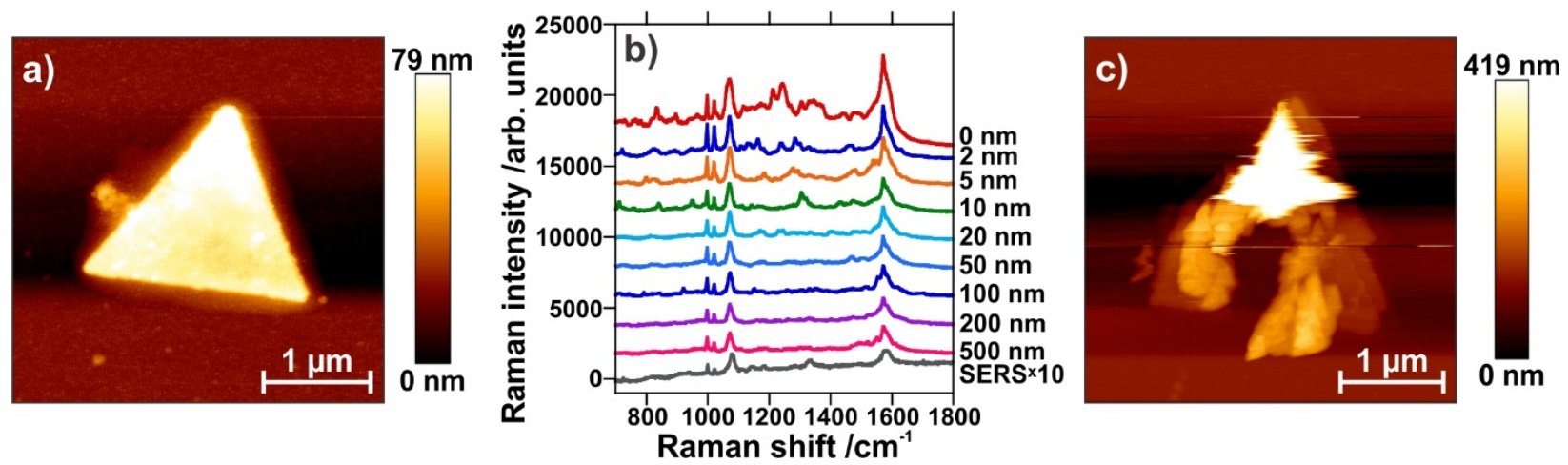

Figure 2: a) AFM of silver nanoplate; b) TERS spectra obtained upon increasing the tip-sample distance, as indicated, compared to the SERS spectrum multiplied by 10. Spectra have been offset for clarity; c) AFM of silver nanoplate after TERS experiment.
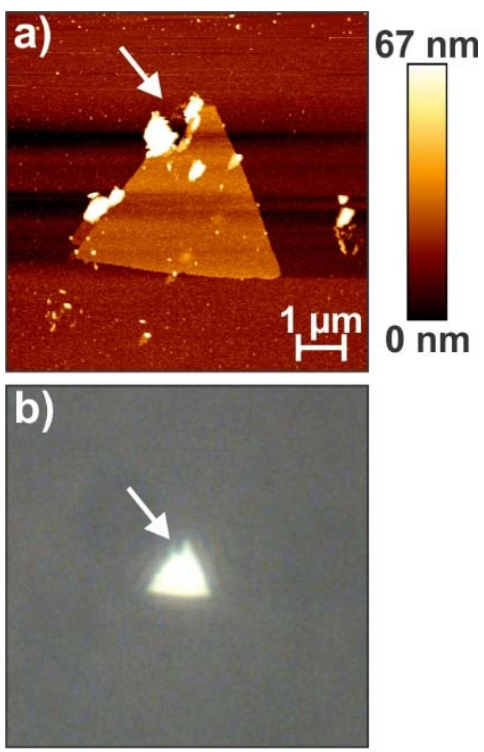

Figure 3: a) Atomic force micrograph of a non-functionalized drilled silver nanoplate, with the arrow indicating damage; b) Image of the same nanoplate, with the arrow indicating the same point. 
To further investigate the cause of this localized destruction, a TERS experiment was performed under optical misalignment, where the tip was placed on the edge of the focused laser focal plane. Under such a configuration, little to no electromagnetic enhancement at the tip apex should be present. As depicted in Figure $4 \mathrm{a}$ and $4 \mathrm{~b}$, selected points for spectral collection were chosen on and off the nanoplate. Under the misaligned conditions, no TERS spectra of the adsorbed molecule could be collected (Figure 4c). As the irradiation time for this experiment was significantly shorter than that used in Figure 2, the sample was exposed to the laser for an additional 5 minutes to determine if the nanoplate had been damaged by laser-induced heating. The post-irradiation AFM scan (Figure 4d) did not exhibit any damage. To verify that the nanoplate was not impervious to drilling, the tip and laser were realigned to be in an ideal TERS configuration. Spectra were collected on and off the nanoplate (red and blue dots, Figure 4a), and the comparison in Figure 4e shows that the system is aligned, as the 4-MPBA signal is seen on, but not off, the nanoplate. Ten further spectra were collected in the centre of the nanoplate, at varying tip-sample distances (Figure S1). Additional single point measurements were collected at other positions on the nanoplate (Figure 4b). After collection of the TERS spectra in the selected areas, a subsequent AFM scan was acquired (Figure 4f). Once again, local damage was observed in the exact position where the spectra were collected. The defects exhibit two critical structural components. In the region that was irradiated over a longer period (barycenter of the triangle), the degree of damage is considerably greater than that of the areas where single TERS measurements were performed. Furthermore, regardless of the exposure time, the defects adapt a triangular shape, similar to that of the AFM tip. As the positions and relative sizes of the holes can be readily changed based on the TERS experiments, this approach could be used to pattern nanostructures through localized metal ablation. 

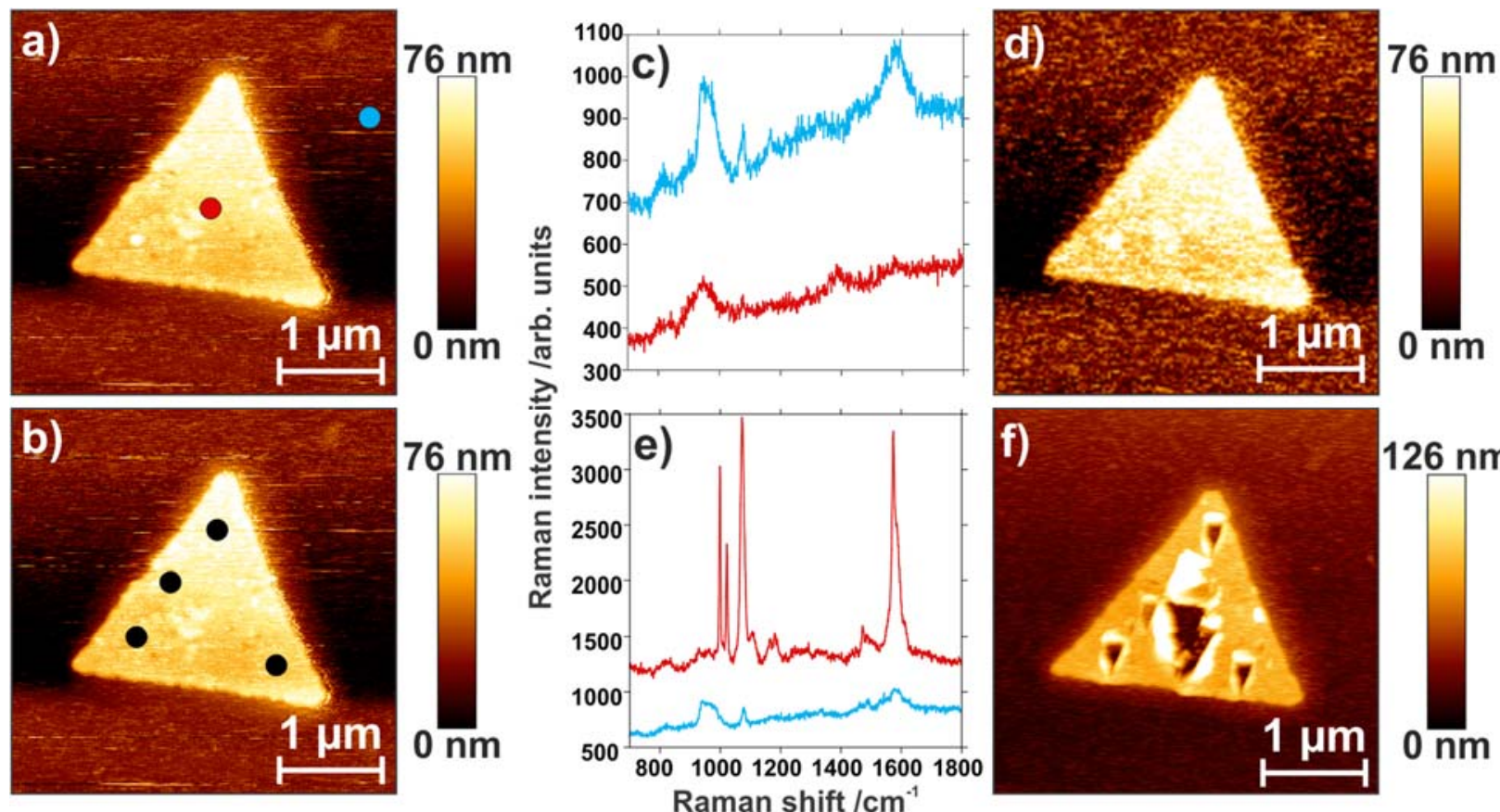

$126 \mathrm{~nm}$

Figure 4: Atomic force scans of silver nanoplate, showing a) points at which spectra were collected on (red) and off (blue) the nanoplate, and b) other points of collection. c) No TERS signal is measured off alignment, resulting in d) no damage to the nanoplate. e) TERS spectrum is measured on the nanoplate (red), resulting in f) damage to the nanoplate.

These observations indicate that under an ideal TERS configuration, the TERS tip can induce significant damage on a metallic surface, and can be used to drill triangular holes into the nanoplates. As metallic nanoplates are commonly used in gap-mode TERS measurements, the source of this nanoscale surface ablation was further investigated. Possible causes of this drilling are multiple and can presumably be assigned to i) mechanical damage from the tip; ii) plasmonic heating; iii) a plasmon-mediated chemical or electrochemical reaction; or iv) some combination of these processes. As mentioned earlier, no hole generation was observed when the laser was not focused on the tip. Since pseudo-TERS measurements were performed (single point and tipretraction), it is unlikely that mechanical damage is the cause, despite that triangular shape matching that of the metallized AFM tips. Furthermore, under prolonged irradiation with this 
configuration, no damage was observed. This means that laser heating from a far-field focused beam alone is insufficient to drive this process, and that the presence of the tip at the focal point of the laser is necessary to induce local damage. The excitation source was set at $632.8 \mathrm{~nm}$, and since the plasmon excitation is quite weak at this wavelength, this may exclude thermal effects in these experimental conditions. This does not preclude the possibility of heating due to the plasmonic resonance of the tip. As such, we focus here on the effect of an ideal TERS configuration, where the metallized tip is aligned with the laser.

\section{Heat Generation at the TERS Tip}

Optical confinement of the electric field in small volumes of metal nanoparticles results in plasmonic heating. ${ }^{46}$ This heating been exploited, among other uses, for cancer phototherapy. ${ }^{47-48}$ Plasmonic heating can be sufficient to vaporize the surrounding medium, ${ }^{49-50}$ or under intense continuous wave sources,${ }^{51}$ or pulsed lasers,${ }^{52}$ locally melt the nanoparticle. Previous studies have shown plasmonic heating to adversely affect the SERS spectra, as molecules are desorbed or nanoparticles are damaged. ${ }^{53-54}$ To investigate the heating of the tip, finite-element method (FEM) calculations were performed to evaluate the temperature gradient in the vicinity of the tipnanoplate assembly. The geometry and material properties were set to match the experiment, with the laser linearly polarized and propagating along the tip axis. The normalized electric field was calculated for a tip-sample separation of $2 \mathrm{~nm}$, as shown in Figure 5a. The corresponding temperature change is reported in Figure 5b. For a tightly focused Gaussian beam, there is a component of the electric field along the tip axis; however, it is difficult to ascertain the exact value of this component. To determine the maximum electric field, and thus the maximum heating expected, the case where the laser is polarized along the tip axis with a side illumination geometry is also calculated (Figure S2). 


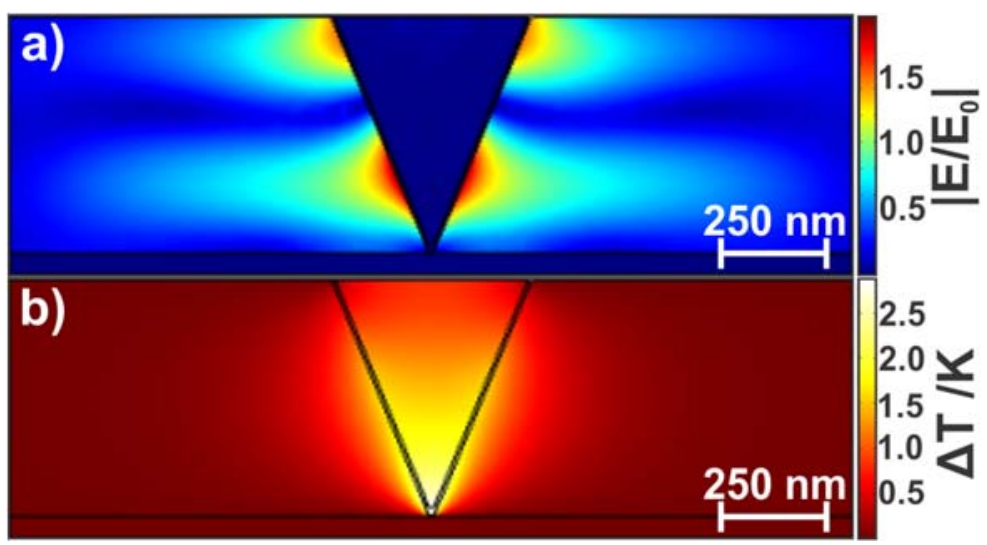

Figure 5: a) Electric field in the plane of polarization, with $2 \mathrm{~nm}$ separation from the silver nanoplate; b) Resultant temperature increase.

This result for the electric field calculation (Figure 5a) is consistent with previous results. ${ }^{23,55}$ The resultant weak temperature increase, limited to about $2 \mathrm{~K}$ as shown in Figure $5 \mathrm{~b}$, is comparable to the work of Balois et al., who have predicted an increase of several degrees under $50 \mu \mathrm{W}$ plane wave side illumination with a gold tip-gold substrate junction. ${ }^{55}$ Other work from Downes et al. predicts a much larger temperature increase, up to $\Delta \mathrm{T}=360 \mathrm{~K}$ in the gold tip-silver substrate junction at $533 \mathrm{~nm}$ using side illumination; ${ }^{56}$ in comparison, the reciprocal metal configuration (Ag tip and Au substrate) illuminated at $633 \mathrm{~nm}$ increases by only $30 \mathrm{~K}$ under the same irradiance conditions of $1 \mathrm{~mW} / \mu^{2}{ }^{2}{ }^{57}$ Differences between these results and ours can be attributed to distinct excitation wavelengths and geometry. In the backscattering configuration that we have used for our experiment and modelling, it is likely that the metal substrate has decreased the laser intensity reaching the tip, leading to limited heating at the tip apex as observed in Figure 5b. As shown in Figure $\mathrm{S} 2$, polarization along the tip axis yields a higher temperature increase of $\Delta \mathrm{T} \sim 25 \mathrm{~K}$ but, to reiterate, the difference between our study and that of Downes et al. is their illumination with $532 \mathrm{~nm}$ compared to our excitation with $632.8 \mathrm{~nm}$, as both calculations were conducted with 
irrandiances of $1 \mathrm{~mW} / \mu \mathrm{m}^{2}$. The FEM simulations show that the calculated temperatures at the tipsubstrate junction are insufficient to cause drastic damage to the nanoplates.

\section{Plasmon-Mediated Reactions at a TERS Tip}

To further investigate the role of the tip in the nanoplate drilling, the TERS tip was investigated under SEM and energy-dispersive X-ray (EDX) spectroscopy. SEM images were first obtained for a pristine TERS tip (Figure 6a,b). A tip coated at the same time as the pristine tip was used for the TERS experiment. The tip selected for EDX measurements was minimally used. In this instance, the laser was focused on the nanoplate. When the tip was raster scanned over the nanoplate during the laser-tip alignment, the tip drilled a hole near the middle of the nanoplate (Figure S3). As this tip was not in the focal spot of the laser for only a few seconds, and was in motion during this time, plasmon-induced heating was minimized.

Figure $6 \mathrm{c}$ and $\mathrm{d}$ show the damage to the tip after the TERS experiment. As seen in Figure $6 \mathrm{~d}$, the gold layer is delaminated from the tip, revealing the silicon that constitutes the bulk of the tip. There are two distinct sections chosen for EDX measurement, just below and above the tip apex, as highlighted in Figure 6d. In the area above the tip apex, EDX measurements show the expected gold and silicon (Figure 6e): this section is the gold layer, ripped away from the tip during alignment. On the lower section, peaks from silver are also seen (Figure 6f). 

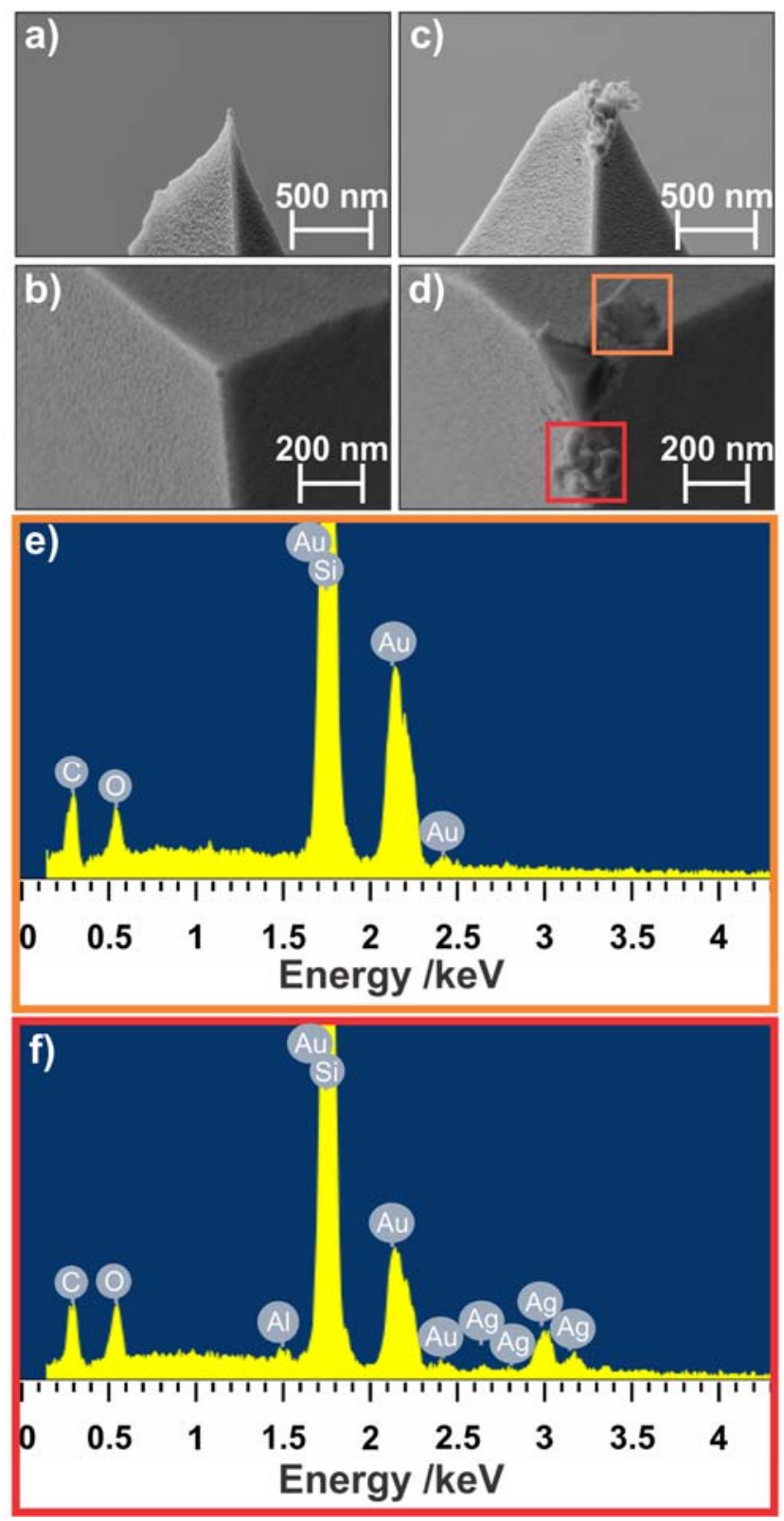

Figure 6: Scanning electron micrographs of a), b) a pristine TERS tip; c) a TERS tip after silver nanoplate drilling, with d) areas at which EDX spectra were collected; e), f) corresponding EDX spectra.

Maps showing the distribution of each element of interest were also acquired, correlating to the SEM of the damaged tip (Figure 7a). The gold map, in Figure 7b, shows gold in all undamaged areas of the tip, but not the exposed silicon or the area below the tip apex. Conversely, the silicon 
map shows the highest signal in the area exposed (Figure 7c), is also present under the undamaged layer of gold, but no signal is seen in the area below the tip apex. The map of silver shows only a presence in the area below the tip apex (Figure 7d). Gold and silicon are also likely present under the tip apex, but are shielded by the silver structure.
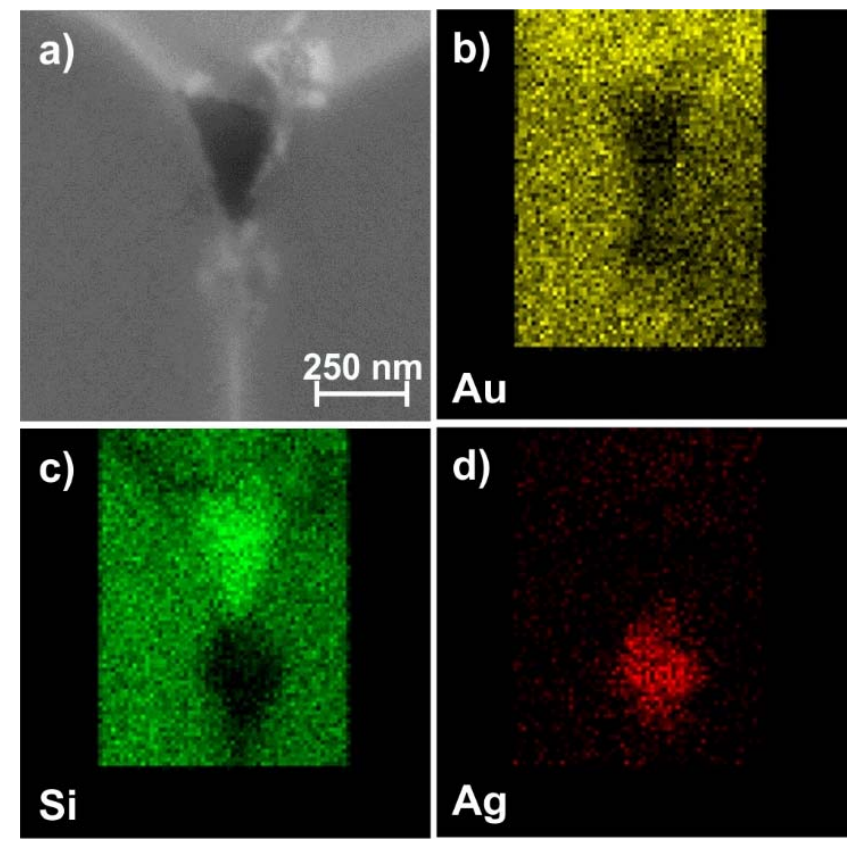

Figure 7: a) Scanning electron micrograph of a tip after drilling; EDX maps of b) gold; c) silicon; d) silver.

The presence of amorphous silver on the TERS tip suggests silver species are transferred from the nanoplate to the tip by a plasmon-mediated reaction. Such reactions are triggered by the combination of the locally enhanced electric field and the presence of hot electrons, highly energetic electrons that are the result of non-radiative plasmon decay. The generation of hot electrons has been described for both silver and gold nanostructures. To further understand the relationship between TERS and hot-electron generation, it is necessary to compare our configuration to other gap-mode TERS experiments. 
Typically, gap-mode TERS experiments employ the use of gold nanoplates. For the resulting tip-plate junctions (gold-gold, silver-gold), to the best of our knowledge, no previous studies have demonstrated deformation of the nanoplate after TERS measurements to the degree we have shown and under modest irradiation conditions. The use of a silver nanoplate with a gold tip is likely the driving force behind our 2D patterning of a metallic surface. Here, we surmise that two factors either individually or in combination, yield the changes to the structure of the nanoplate. It has been shown the citrate can be oxidized by hot holes, which can then reduce $\mathrm{Ag}^{+}$ions. ${ }^{58}$ Under different conditions, $\mathrm{Ag}^{+}$can be reduced directly by hot electrons. ${ }^{40}$ The transfer of the hot electrons from the tip to the nanoplate can be aided by the layer of atmospheric water at the tipsubstrate interface, or from the direct contact between the tip and nanoplate during measurement. As citrate is used in the synthesis of the nanoplates described in this study, it is possible that even after functionalization with 4-MPBA, some citrate remains on the surface. It is known that coating a surface with a thin layer of $\mathrm{SiO}_{2}$ can prevent hot electrons from reaching or escaping the surface. ${ }^{59}$ However, in the case of coating the metal tip with such a layer, we have previously shown that this diminishes the EM enhancement and resulting TERS compatibility of the tip. ${ }^{23}$ As the use of silver nanoplates with gap-mode TERS is limited, ${ }^{15-16}$ it is difficult to ascertain if this is effect occurs exclusively with silver nanoplates. In those described works, a silver tip was used in conjunction with the silver plates. However, we employ the use of a gold tip as opposed to a silver tip. As our conditions use a heterometallic junction, the hot electron generated by the gold tip could reduce surface silver species onto the tip, potentially when the tip makes contact with the surface. The triangular geometry of the resulting hole is related to the direction of the hot electron coming off of the tetrahedral tip. 


\section{CONCLUSIONS}

In summary, silver nanoplates were synthesized by a seed-mediated method for use as substrates for gap-mode TERS experiments. During both the alignment process and the collection of TERS spectra, nanoscale holes with a triangular shape were ablated in the nanoplate when the tip was aligned with the laser. Using FEM calculations, we investigated the role of plasmon-induced heating, and determined that the elevation in temperature at the junction between the tip and nanoplate was not enough to account for the surface damage. EDX measurements of a tip used to generate the triangular nanoholes showed the presence of silver on the tip, making the reduction of silver species from the nanoplate onto the tip a likely cause of this nanoplate drilling. Further investigations to determine the exact trigger of this process could provide greater insight into plasmon-mediated chemical reactions, especially when they can be controlled with nanoscale precision. Additionally, as this study has shown, the nanoscale patterning of $2 \mathrm{D}$ materials can expand beyond reactions involving the surface species but can be used to prepare metal surfaces with $2 \mathrm{D}$ patterns. These patterned metasurfaces would exhibit unique optical properties compared to the original nanoplate, such as improved SERS compatibility.

\section{METHODS}

Silver nanoplate synthesis. A seed solution was prepared by combining aqueous $\mathrm{AgNO}_{3}(0.1$ mM, $25 \mathrm{~mL})$, trisodium citrate (30 mM, $0.3 \mathrm{~mL})$, PVP (MW $29000 \mathrm{~g} / \mathrm{mol}, 3.5 \mathrm{mM}, 1.5 \mathrm{~mL})$ and $\mathrm{H}_{2} \mathrm{O}_{2}(30 \mathrm{wt} \%, 60 \mu \mathrm{L})$ under stirring. An aqueous $\mathrm{NaBH}_{4}$ solution $(100 \mathrm{M}, 250 \mu \mathrm{L})$ was injected, and the solution was left to stir for $30 \mathrm{~min}$. The solution was centrifuged at $11000 \mathrm{rpm}$ for $8 \mathrm{~min}$, and the nanoplates were redispersed in deionized water. This washing step was repeated twice, and the seeds were then dispersed in $40 \mathrm{~mL}$ of deionized water. In the seeded growth process, Lascorbic acid $(0.1 \mathrm{M}, 0.375 \mathrm{~mL})$ and trisodium citrate $(0.075 \mathrm{M}, 0.125 \mathrm{~mL})$ were injected to 10 $\mathrm{mL}$ of the seed solution under stirring. A solution of $\mathrm{AgNO}_{3}(1 \mathrm{mM}, 20 \mathrm{~mL})$, citric acid $(0.1 \mathrm{M}$, 
$0.125 \mathrm{~mL})$, and sodium citrate $(1.5 \mathrm{mM}, 0.1 \mathrm{~mL})$ was prepared. It was added to the seed solution using a syringe pump, at a rate of $0.2 \mathrm{~mL} / \mathrm{min}$. After injection, $20 \mathrm{~mL}$ of the reaction solution was removed, and the remaining solution was used as seeds for the next growth cycle. In total, four rounds of growth were performed. Nanoplates were dropcasted onto clean coverslips.

Self-assembled monolayer preparation. The nanoplate sample was functionalized with 4mercaptophenyl boronic acid (4-MPBA). 4-MPBA was dissolved in hot ethanol to produce a $10^{-}$

${ }^{3} \mathrm{M}$ solution. The nanoplate sample was immersed in the solution for $24 \mathrm{~h}$. The sample was then rinsed in ethanol, to remove any non-covalently-bonded 4-MPBA, and dried under nitrogen.

TERS tip preparation. Commercial AFM tips (NCL-50, NanoWorld) were prepared for TERS by electron-beam evaporation. An adhesion layer of $5 \mathrm{~nm}$ of Ti was deposited, followed by $20 \mathrm{~nm}$ of Au. The deposition rate was $0.3-0.5 \AA / \mathrm{s}$.

TERS set-up. The TERS set-up combines a Raman spectrometer (HR LabRam, Horiba) connected to an inverted optical microscope (Olympus IX71) with an AFM (NanoWizard II, JPK Instruments). Measurements are performed in transmission mode, such that light is focused from below onto the metallized tip and the sample. A $\times 100$ objective (NA 0.9) (PlanAPO Olympus) objective focused the laser on the sample and collected the Raman signal. The Raman signal is detected using a liquid nitrogen-cooled charge-coupled device (Horiba, Symphony), with the fundamental laser light removed by a notch filter. A photodiode is connected to the AFM control so that Rayleigh scattering from the TERS tip can be measured to align the tip in the focal point of the objective.

Using an excitation laser of $632.8 \mathrm{~nm}$ with an intensity of $1 \mathrm{~mW}$ at the sample, the Rayleigh scattering from the metallized tip was measured by the photodiode. After aligning the tip at the 
centre of the focal point, the tip was frozen in the $x$ - and $y$-positions. An AFM scan was obtained by sample scanning.

The TERS signal was collected at points with a $600 \mathrm{gr} / \mathrm{mm}$ grating and a spectrometer slit width of $200 \mu \mathrm{m}$. Spectra were typically recorded in the $700-1800 \mathrm{~cm}^{-1}$ range with a $10 \mathrm{~s}$ acquisition time per spectrum.

Finite-element modelling. COMSOL Multiphysics 5.2 was employed for finite-element modelling of the electric field and resulting temperature. The TERS tip was modelled as an inverted Si cone with an angle of $22.5^{\circ}$, with a spherical tip $30 \mathrm{~nm}$ in diameter and a $20 \mathrm{~nm}$ thick Au shell. The geometry is $2 \mu \mathrm{m}$ wide, and $600 \mathrm{~nm}$ tall. In air, the tip is positioned above the silver nanoplate, which is $50 \mathrm{~nm}$ thick. The geometry is meshed tetrahedrally, with an element size $2-$ $50 \mathrm{~nm}$, with a growth rate of 1.45 and curvature factor of 0.5 . The material properties are summarized in Table S1. Where necessary, the relative permeability was set as 1 . Initially, there is no electric field and the temperature is $293.15 \mathrm{~K}$. All boundaries are held at room temperature (293.15 K), except the top of the tip, which is thermally insulated. Perfectly matched layers (PML) of $200 \mathrm{~nm}$ are set at edges of the geometry, to absorb reflections. The PML was meshed using a swept mesh, with 8 layers. A scattering boundary condition is applied along the top face of the air boundary. The bottom of the geometry is illuminated by a tightly-focused Gaussian beam at 632.8 $\mathrm{nm}, 1 \mu \mathrm{m}$ spot size and $1 \mathrm{~mW}$ of power, to match experimental values. As the Gaussian beam was tightly focused, the longitudinal component of the electric field was set as: ${ }^{60}$

$$
E_{z}=i k_{0} \frac{\partial E_{x}}{\partial x}
$$

Using the Multiphysics approach, the electric field is calculated using the Wave Optics module, and is linked to the Heat Transfer. 
Tip characterization. Scanning Electron Microscope (SEM) images of TERS tips were obtained using a LEO Zeiss 1540XB (Zeiss, Oberkochen, Germany), fitted with an Oxford Instruments X-ray system, allowing for in situ EDX analyses and mapping.

\section{ASSOCIATED CONTENT}

Supporting Information. Additional TERS spectra; simulated heating in side illumination geometry; AFM of damaged nanoplate; calulation details

\section{AUTHOR INFORMATION}

\section{Corresponding Author}

* E-mail address: flagugne@uwo.ca (F.L-L.)

\section{Author Contributions}

The manuscript was written through contributions of all authors. All authors have given approval to the final version of the manuscript.

\section{Notes}

The authors declare no competing financial interests.

\section{ACKNOWLEDGMENT}

The authors gratefully acknowledge the Nanofabrication Facility at Western University; in particular, Dr. Todd Simpson, for assistance with the SEM and EDX measurements. The authors gratefully acknowledge Fraser Filice for his many insightful discussions regarding the simulations. This research was supported by the Natural Sciences and Engineering Research 
Council (NSERC) of Canada. This research was further funded by the Canada Research Chairs

program in "Photonics and Nanosciences" (F.L.-L.). D.M.M. thanks the Ontario Graduate

Scholarship program.

\section{REFERENCES}

1. Al-Shammari, R. M.; Al-attar, N.; Manzo, M.; Gallo, K.; Rodriguez, B. J.; Rice, J. H., Single-Molecule Nonresonant Wide-Field Surface-Enhanced Raman Scattering from Ferroelectrically Defined Au Nanoparticle Mircoarrays. ACS Omega 2018, 3, 3165-3172.

2. de Albuquerque, C. D. L.; Sobral-Filho, R. G.; Poppi, R. J.; Brolo, A. G., Digital Protocol for Chemical Analysis at Ultralow Concentrations by Surface-Enhanced Raman Scattering. Anal. Chem. 2018, 90, 1248-1254.

3. Sprague-Klein, E. A.; McAnally, M. O.; Zhdanov, D. V.; Zrimsek, A. B.; Apkarian, V. A.; Seieman, T.; Schatz, G. C.; Van Duyne, R. P., Observation of Single Molecule PlasmonDriven Electron Transfer in Isotopically Edited 4,4'-Bipyridine Gold Nanosphere Oligomers. $J$. Am. Chem. Soc. 2017, 139, 15212-15221.

4. $\quad$ Limaj, O.; Etezadi, D.; Wittenberg, N. J.; Rodrigo, D.; Yoo, D.; Oh, S.-H.; Altug, H., Infrared Plasmonic Biosensor for Real-Time and Label-Free Monitoring of Lipid Membranes. Nano Lett. 2016, 16, 1502-1508.

5. Vogt, J.; Zimmerman, S.; Huck, C.; Tzschoppe, M.; Neubrech, F.; Fatikow, S.; Pucci, A., Chemical Identification of Individual Fine Dust Particles with Resonant Plasmonic Enhancement of Nanoslits in the Infrared. ACS Photonics 2017, 4, 560-566.

6. Wallace, G. Q.; Read, S. T.; McRae, D. M.; Rosendahl, S. M.; Lagugné-Labarthet, F., Exploiting Anisotropy of Plasmonic Nanostructures with Polarization Modulation Infrared Linear Dichroism Microscopy ( $\mu$ PM-IRLD). Adv. Opt. Mater. 2018, 6, 1701336.

7. Touzalin, T.; Joiret, S.; Maisonhaute, E.; Lucas, I. T., Complex Electron Transfer Pathway at a Microelectrode Captured by in Situ Nanospectroscopy. Anal. Chem. 2017, 89, 8974-8980.

8. Awada, C.; Plathier, J.; Dab, C.; Charra, F.; Douillard, L.; Ruediger, A., High Resolution Scanning Near Field Mapping of Enhancement on SERS Substrates: Comparison with Photoemission Electron Microscopy. Phys. Chem. Chem. Phys. 2016, 18, 9405-9411.

9. $\quad$ Pashaee, F.; Hou, R.; Gobbo, P.; Workentin, M. S.; Lagugné-Labarthet, F., Tip-Enhanced Raman Spectroscopy of Self-Assembled Thiolated Monolayers on Flat Gold Nanoplates Using Gaussian-Transverse and Radially Polarized Excitations. J. Phys. Chem. C 2013, 117, 1563915646.

10. Kumar, N.; Stephanidis, B.; Wain, A. J.; Roy, D., Nanoscale Mapping of Catalytic Activity Using Tip-Enhanced Raman Spectroscopy. Nanoscale 2015, 7, 7133-7137.

11. Stadler, J.; Oswald, B.; Schmid, T.; Zenobi, R., Characterizing Unusual Metal Substrates for Gap-Mode Tip-Enhanced Raman Spectroscopy. J. Raman Spectrosc. 2012, 44, 227-233. 12. van Schrojenstein Lantman, E. M.; Deckert-Gaudig, T.; Mank, A. J. G.; Deckert, V.; Weckhuysen, B. M., Catalytic Processes Monitored at the Nanoscale with Tip-Enhanced Raman Spectroscopy. Nat. Nanotechnol. 2012, 7, 583-586.

13. Alexander, K. D.; Schultz, Z. D., Tip-Enhanced Raman Detection of Antibody Conjugated Nanoparticles on Cellular Membranes. Anal. Chem. 2012, 84, 7408-7414. 
14. Blum, C.; Schmid, T.; Opilik, L.; Metanis, N.; Weidmann, S.; Zenobi, R., Missing Amide I Mode in Gap-Mode Tip-Enhanced Raman Spectra of Proteins. J. Phys. Chem. C 2012, 116, 23061-23066.

15. Deckert-Gaudig, T.; Deckert, V., Tip-Enhanced Raman Scattering Studies of Histidine on Novel Silver Substrates. J. Raman Spectrosc. 2009, 40, 1446-1451.

16. Deckert-Gaudig, T.; Erver, F.; Deckert, V., Transparent Silver Microcrystals: Synthesis and Application for Nanoscale Analysis. Langmuir 2009, 25, 6032-6034.

17. Deckert-Gaudig, T.; Kämmer, E.; Deckert, V., Tracking of Nanoscale Structural Variations on a Single Amyloid Fibril with Tip-Enhanced Raman Scattering. J. Biophotonics 2011, 5, 215-219.

18. Deckert-Gaudig, T.; Rauls, E.; Deckert, V., Aromatic Amino Acid Monolayers Sandwiched between Gold and Silver: A Combined Tip-Enhanced Raman and Theoretical Approach. J. Phys. Chem. C 2010, 114, 7412-7420.

19. Pashaee, F.; Tabatabaei, M.; Caetano, F. A.; Ferguson, S. S. G.; Lagugné-Labarthet, F., Tip-Enhanced Raman Spectroscopy: Plasmid-Free vs. Plasmid-Embedded DNA. Analyst 2016, 141, 3251-3258.

20. Paulite, M.; Blum, C.; Schmid, T.; Opilik, L.; Eyer, K.; Walker, G. C.; Zenobi, R., Full Spectroscopic Tip-Enhanced Raman Imaging of Single Nanotapes Formed from $\beta$-Amyloid (140) Peptide Fragments. ACS Nano 2013, 7, 911-920.

21. Sereda, V.; Lednev, I. K., Two Mechanisms of Tip Enhancement of Raman Scattering by Protein Aggregates. Appl. Spectrosc. 2016, 7, 118-128.

22. Deckert-Gaudig, T.; Deckert, V., Ultraflat Transparent Gold Nanoplates - Ideal Substrates for Tip-Enhanced Raman Scattering Experiments. Small 2009, 5, 432-436.

23. Kazemi-Zanjani, N.; Vedraine, S.; Lagugné-Labarthet, F., Localized Enhancement of Electric Field in Tip-Enhanced Raman Spectroscopy Using Radially and Linearly Polarized Light. Opt. Express 2013, 21, 25271-25276.

24. Brown, A. M.; Sundaraman, R.; Narang, P.; Goddard, W. A., III; Atwater, H. A., Nonradiative Plasmon Decay and Hot Carrier Dynamics: Effects of Phonons, Surfaces, and Geometry. ACS Nano 2016, 10, 957-966.

25. Manjavacas, A.; Liu, J. G.; Kulkarni, V.; Nordlander, P., Plasmon-Induced Hot Carriers in Metallic Nanoparticles. ACS Nano 2014, 8, 7630-7638.

26. Brooks, J. L.; Frontiera, R. R., Competition between Reaction and Degradation Pathways in Plasmon-Driven Photochemistry. J. Phys. Chem. C 2016, 120, 20869-20876.

27. Dong, B.; Fang, Y.; Chen, X.; Xu, H.; Sun, M., Substrate-, Wavelength-, and TimeDependent Plasmon-Assisted Surface Catalysis Reaction of 4-Nitrobenzenethiol Dimerizing to p, p'-Dimercaptoazobenzene on Au, Ag, and Cu Films. Langmuir 2011, 27, 10677-10682.

28. Huang, Y.-F.; Zhu, H.-P.; Liu, G.-K.; Wu, D.-Y.; Ren, B.; Tian, Z.-Q., When the Signal Is Not from the Original Molecule To Be Detected: Chemical Transformation of paraAminothiophenol on Ag during the SERS Measurement. J. Am. Chem. Soc. 2010, 132, 92449246.

29. Liebig, F.; Sarhan, R. M.; Sander, M.; Koopman, W.; Schuetz, R.; Bargheer, M.; Koetz, J., Deposition of Gold Nanotriangles in Large Scale Close-Packed Monolayers for X-ray-Based Temperature Calibration and SERS Monitoring of Plasmon-Driven Catalytic Reactions. ACS Appl. Mater. Interfaces 2017, 9, 20247-20253. 
30. Xu, P.; Kang, L.; Mack, N. H.; Schanze, K. S.; Han, X.; Wang, H.-L., Mechanistic Understanding of Suface Plasmon Assisted Catalysis on a Single Particle: Cyclic Redox of 4Aminothiophenol. Sci. Rep. 2013, 3, 2997.

31. Zhang, Z.; Richard-Lacroix, M.; Deckert, V., Plasmon Induced Polymerization Using a TERS Approach: A Platform for Nanostructured 2D/1D Material Production. Faraday Discuss. 2017, 2015, 213-226.

32. Nguyen, M.; Lamouri, A.; Salameh, C.; Lévi, G.; Grand, J.; Boubekeur-Lecaque, L.; Mangeney, C.; Félidj, N., Plasmon-Mediated Chemical Surface Functionalization at the Nanoscale. Nanoscale 2016, 8, 8633-8640.

33. Zhou, X.; Deeb, C.; Kostcheev, S.; Wiederrecht, G. P.; Adam, P.-M.; Béal, J.; Plain, J.; Gosztola, D. J.; Grand, J.; Félidj, N.; Wang, H.; Vial, A.; Bachelot, R., Selective Functionalization of the Nanogap of a Plasmonic Dimer. ACS Photonics 2015, 2, 121-129. 34. Nguyen, V.-Q.; Ai, Y.; Martin, P.; Lacroix, J.-C., Plasmon-Induced Nanolocalized Reduction of Diazonium Salts. ACS Omega 2017, 2, 1947-1955.

35. Tesema, T. E.; Kafle, B.; Tadesse, M. G.; Habteyes, T. G., Plasmon-Enhanced Resonant Excitation and Demethylation of Methylene Blue. J. Phys. Chem. C 2017, 121, 7421-7428.

36. Lee, J.; Mubeen, S.; Ji, X.; Stucky, G. D.; Moskovits, M., Plasmonic Photoanodes for Solar Water Splitting with Visible Light. Nano Lett. 2012, 12, 5014-5019.

37. Li, H.; Li, Z.; Yu, Y.; Ma, Y.; Yang, W.; Wang, F.; Yin, X.; Wang, X., Surface-PlasmonResonance-Enhanced Photoelectrochemical Water Splitting from Au-Nanoparticle-Decorated 3D TiO2 Nanorod Architectures. J. Phys. Chem. C 2017, 121, 12071-12079.

38. Liu, Z.; Hou, W.; Pavaskar, P.; Aykol, M.; Cronin, S. B., Plasmon Resonant Enhancement of Photocatalytic Water Splitting Under Visible Illumination. Nano Lett. 2011, 11, 1111-1116.

39. Kim, N. H.; Meinhart, C. D.; Moskovits, M., Plasmon-Mediated Reduction of Aqueous Platinum Ions: The Competing Roles of Field Enhancement and Hot Charge Carriers. J. Phys. Chem. C 2016, 120, 6750-6755.

40. Lee, S. J.; Piorek, B. D.; Meinhart, C. D.; Moskovits, M., Photoreduction at a Distance: Facile, Nonlocal Photoreduction of Ag Ions in Solution by Plasmon-Mediated Photoemitted Electrons. Nano Lett. 2010, 10, 1329-1334.

41. Sun, M.; Zhang, Z.; Zheng, H.; Xu, H., In-Situ Plasmon-Driven Chemical Reactions Revealed by High Vacuum Tip-Enhanced Raman Spectroscopy. Sci. Rep. 2012, 2, 647.

42. Dab, C.; Awada, C.; Merlen, A.; Ruediger, A., Near-Field Chemical Mapping of Gold Nanostructures Using a Functionalized Scanning Probe. Phys. Chem. Chem. Phys. 2017, 19, 31063-31071.

43. Chu, H.-C.; Kuo, C.-H.; Huang, M. H., Thermal Aqueous Solution Approach for the Synthesis of Triangular and Hexagonal Gold Nanoplates with Three Different Size Ranges. Inorg. Chem. 2005, 45, 808-813.

44. Guo, Z.; Zhang, Y.; Mao, Y.; Huang, L.; Gu, N., Synthesis of Microsized Gold Nanoplates by a Self-Seeding Method in Ethanol Solution. Mater. Lett. 2006, 60, 3522-3525. 45. Zhang, Q.; Hu, Y.; Goebl, J.; Yin, Y., Seeded Growth of Uniform Ag Nanoplates with High Aspect Ratio and Widely Tunable Surface Plasmon Bands. Nano Lett. 2010, 10, 50375042.

46. Baffou, G.; Quidant, R., Thermo-Plasmonics: Using Metallic Nanostructures as NanoSources of Heat. Laser Photonics Rev. 2012, 7, 171-187. 
47. Ricciardi, L.; Sancey, L.; Palermo, G.; Termine, R.; De Luca, A.; Szerb, E. I.; Aiello, I.; Ghedini, M.; Strangi, G.; Le Deda, M., Plasmon-Mediated Cancer Phototherapy: The Combined Effect of Thermal and Photodynamic Processes. Nanoscale 2017, 9, 19279-19289.

48. Lal, S.; Clare, S. E.; Halas, N. J., Nanoshell-Enabled Photothermal Cancer Therapy: Impending Clinical Impact. Acc. Chem. Res. 2008, 41, 1842-1851.

49. Dagallier, A.; Boulais, E.; Boutopoulos, C.; Lachaine, R.; Meunier, M., Multiscale Modeling of Plasmonic Enhanced Energy Transfer and Cavitation around Laser-Excited Nanoparticles. Nanoscale 2017, 9, 3023-3032.

50. Baffou, G.; Polleux, J.; RIgneault, H.; Monneret, S., Super-Heating and Micro-Bubble Generation around Plasmonic Nanoparticles under cw Illumination. J. Phys. Chem. C 2014, 118, 4890-4898.

51. Setoura, K.; Okada, Y.; Hashimoto, S., CW-Laser-Induced Morphological Changes of a Single Gold Nanoparticle on Glass: Observation of Surface Evaporation. Phys. Chem. Chem. Phys. 2014, 16, 26938-26945.

52. Valev, V. K.; Denkova, D.; Zheng, X.; Kuznetsov, A. I.; Reinhardt, C.; Chichkov, B. N.; Tsutsumanova, G.; Oslet, E. J.; Petkov, V.; De Clercq, B.; Silhanek, A. V.; Jeyaram, Y.; Volskiy, V.; Warburton, P. A.; Vandenbosch, G. A. E.; Russev, S.; Aktsipetrov, O. A.; Ameloot, M.; Moschchalkov, V. V.; Verbiest, T., Plasmon-Enhanced Sub-Wavelength Laser Ablation: Plasmonic Nanojets. Adv. Mater. 2012, 24, 29-35.

53. Zeng, Z.-C.; Wang, H.; Johns, P.; Hartland, G. V.; Schultz, Z. D., Photothermal Microscopy of Coupled Nanostructures and the Impact of Nanoscale Heating in SurfaceEnhanced Raman Spectroscopy. J. Phys. Chem. C 2017, 121, 11623-11631.

54. Mochizuki, M.; Lkhamsuren, G.; Suthiwanich, K.; Mondarte, E. A.; Yano, T.; Hara, M.; Hayashi, T., Damage-Free Tip-Enhanced Raman Spectroscopy for Heat-Sensitive Materials. Nanoscale 2017, 9, 10715-10720.

55. Balois, M. V.; Hayazawa, N.; Catalan, F. C.; Kawata, S.; Yano, T.; Hayashi, T., TipEnhanced THz Raman Spectroscopy for Local Temperature Determination at the Nanoscale. Anal. Bioanal. Chem. 2015, 407, 8205-8213.

56. Downes, A.; Salter, D.; Elfick, A., Heating Effects in Tip-Enhanced Optical Microscopy. Opt. Express 2006, 14, 5216-5222.

57. Zhang, W.; Schmid, T.; Yeo, B.-S.; Zenobi, R., Near-Field Heating, Annealing, and Signal Loss in Tip-Enhanced Raman Spectroscopy. J. Phys. Chem. C 2008, 112, 2104-2108. 58. Xiaojuan, C.; Wen, R.; Zhang, L.; Lahiri, A.; Wang, P.; Fang, Y., Photoreduction of Silver Salts Using Au Nanoparticles to Form a Core-Shell-Type Nanostructure: Insight into the Reaction Mechanism. Plasmonics 2014, 9, 945-949.

59. Takeyasu, N.; Yamaguchi, K.; Kagawa, R.; Kaneta, T.; Benz, F.; Fujii, M.; Baumberg, J. J., Blocking Hot Electron Emission by $\mathrm{SiO}_{2}$ Coating Plasmonic Nanostructures. J. Phys. Chem. C 2017, 121, 18795-18799.

60. Lax, M., From Maxwell to Paraxial Wave Optics. Phys. Rev. A 1975, 11, 1365-1370. 